\title{
Efficacy of a cosmetic product mimicking PRP in androgenetic alopecia
}

\author{
Rinaldi F*, Marzani B, Pinto D and Sorbellini E \\ International Hair Research Foundation, Milan, Italy
}

\begin{abstract}
Introduction: Androgenetic alopecia (AGA) is the most common hair loss disorder. Recently, platelet-rich plasma (PRP) injections have emerged as alternative cell-based therapies for the treatment of AGA. Its efficacy is strictly linked to the release of growth factors (GFs) via alpha-granules degranulation. Among their well-known activity, more recently, GFs are acquiring importance as regards their involvement in the regulation of hair growth cycle. Thanks to the advent of modern biotechnology synthetic polypeptides mimicking growth factors have been developed opening to new therapeutic approaches also in the dermatological field, including hair growth disorder.
\end{abstract}

Objective: The aim of the present study was to evaluate the efficacy of a cosmetic product (TR-M-PRP plus) mimicking PRP composition by means of biomimetic peptides in subjects affected by AGA.

Materials and methods: 60 AGA subjects were treated for three months end evaluated, at the end of the study and after one month of follow-up, as regards hair growth by evaluating total and anagen hair count, anagen/telogen ratio, \% of miniaturization, Hair Mass Index (HMI), and hair shaft diameter.

Results: TR-M-PRP plus treatment produced a statistically significant $(\mathrm{p}<0.001)$ clinical improvement compared with PLACEBO in total and anagen hair counts. The treatment with TR-M-PRP plus resulted in a time- increased improvement in the anagen/telogen ratio, reduction of 5 of miniaturization and increasing of HMI and Hair shaft diameter.

Conclusions: Our study confirms, for the first time, the clinical efficacy of a cosmetic product containing biomimetics peptides (TR-M-PRP plus) mimicking autologous PRP for the treatment of AGA.

\section{Introduction}

Hair loss disorders show high rates of occurrence in men and women. Androgenetic alopecia (AGA) is the most common hair loss disorder, affecting up to $80 \%$ of men and $50 \%$ of women in the course of their life [1-3]. AGA is characterized by a progressive reduction in the hair shaft diameter, length, and pigmentation (miniaturization process). The pathogenesis of AGA is complex with genetic, hormonal, inflammatory, and vascular factors thought to contribute to the onset and progression [1,4-7].

The prevalence of hair loss in the population and its impact on self-esteem and depression [8,9], therapies target to reduce the appearance of thinning by delaying, arresting, or reversing the underlying pathology are highly desirable. Nowadays, the currently approved pharmacotherapies are limited to oral Finasteride, a selective 5 - $\alpha$-reductase inhibitor, and $2 \%$ or $5 \%$ topical Minoxidil solutions or foams [10] neither of which without side effects and inevitably relapse in hair loss when the treatment is halted.

Alternative cell-based therapies for the treatment of AGA have emerged, of which, platelet-rich plasma (PRP) injections appear particularly effective $[11,12]$.

PRP is an autologous product obtained by centrifugation from patients own venous blood with no risk of disease transmission and with a concentration of platelets 4-7 time more than baseline values [13].
The autologous PRP was first used in 1987 by Ferrari et al. [14] to avoid excessive transfusion of homologous blood products after open heart surgery. Since that time, the application of autologous PRP has been safely used and documented in many application fields, such as orthopedics and sports medicine, dentistry, neurosurgery, ophthalmology, urology, and wound healing $[15,16]$.

The rationale of PRP therapy is that an injection of concentrated PRP at sites of injury may affect tissue healing via growth factors released after platelets degranulation [17]. Most abundant growth factors (GFs) are platelet-derived growth factor (PDGF), vascular endothelial growth factor (VEGF), transforming growth factor- $\beta$ (TGF- $\beta$ ), basic fibroblast growth factor (BFGF), epidermal growth factor (EGF), and insulin-like growth factor (IGF). GFs have an important role in cell proliferation, migration, and differentiation [17].

The meta-analysis by Giordano et al. [18] encompassing a total of 194 patients (6 studies) provides compelling evidence about PRP treatment for hair restoration in investigated for AA $[11,19,20]$ even if the majority of research targeted AGA $[21,22]$.

${ }^{\star}$ Correspondence to: Rinaldi Fabio, International Hair Research Foundation, Milan, Italy, Tel: +39-2-76006089 E-mail: fabio.rinaldi@studiorinaldi.com

Key words: PRP, biomimetic peptides, hair loss, AGA

Received: March 01, 2019; Accepted: March 18, 2019; Published: April 03, 2019 
A significantly increased number of hairs per $\mathrm{cm}^{2}$ was observed after treatment with PRP. Furthermore, although the limited number of patients, they found interesting results also in hair cross-section thickness and other secondary outcomes which showed a trend toward benefit.

Anyway, because there is no standardized method for PRP preparation, some differences in product composition might be present. This fact may lead to an altered PRP function, which might explain the controversy found in the literature. Furthermore, differences in PRP composition result from differences in the samples retrieved from person to person. The used end product may vary also by the used volume and the number of injections administered, as well as platelet count, the number or absence of leukocytes, and its protein content [18].

Lately, the use of cosmetic formulation containing biological active ingredients, such as biomimetic peptides, has started to spread not only in consumer skin care but also in the dermatological field.

The aim of this double-blind clinical study was to evaluate the efficacy of a cosmetic product (TR-M-PRP plus) [23] mimicking PRP composition and GFs concentration by means of biomimetic peptides in subjects affected by AGA.

\section{Materials and methods}

\section{Subjects}

A total of 60 subjects were enrolled in this a monocentric randomized study 2 arms of 2 parallel groups (1:1 / 1:1 each) doubleblinded placebo-controlled study. The study was conducted under the approval of the Ethical Independent Committee for Clinical, not pharmacological investigation in Genoa (Italy) and in accordance with the ethical standards of the 1964 Declaration of Helsinki.

\section{Inclusion and exclusion criteria}

Before inclusion, subjects were asked to sign an informed consent form indicating that they are aware of the investigational nature of the study.

Participants to the study were healthy male and female subjects aged 18-60 years suffering from AGA (II-IV Hamilton scale for men and I-II Ludwig scale for women) accepting to not receive any drugs/cosmetics treatment able to interfere with the study results, no participation in a similar study actually or during the previous 6 months.

Exclusion criteria included: known sensitivity to any compound of the Investigational product, pregnant or breastfeeding females or planning a pregnancy, serious intercurrent infection or other active disease up to three months prior to study entry, history of concurrent malignancy, significant concurrent medical disorders that may impair the subject's ability to participate over the whole one year of the study.

\section{Test product}

Subjects were randomized to receive the study product (TRM-PRP plus, Giuliani SpA, Milan) containing biomimetic peptides octapeptide-2, copper tripeptide-1, Oligopeptide-20, acetyl decapeptide-3 -; lactoferrin; lactoglobulin; melatonin; humectant; preservative; viscosity control) or PLACEBO (same formulation without biomimetic peptides, lactoferrin, lactoglobulin, and melatonin).

Subjects were asked to apply $15 \mathrm{ml}$ of TR-M-PRP plus /week (application time at least $5 \mathrm{~h}$ ) or PLACEBO for 3 months. Subjects were asked to apply TR-M-PRP on the entire scalp by gently massaging until absorption. All information has been provided in the form of written recommendations.

\section{Evaluations}

Evaluations were performed at the Baseline Visit (T0) and at the End of Treatment Visit (T1), and at the Follow Up Visit (T2). The TrichoScan software (v. 3.0) (Trichology GmbH, Freiburg, Germany) was employed for the analysis of following hair measurements: total hair count (in a $1 \mathrm{~cm} 2$ area), anagen hair count and anagen/telogen ratio and miniaturization. Hair Mass Index (HMI) was assessed by HairCheck measuring system (Divi International Co., Miami, FL, USA) [24,25].

The Hair check cartridge was first calibrated and then placed in the J-slot and HMI measured. Epiluminescence microscopy (Molemax HD, Derma Instrument, Vienna, Austria) was used for determination of hair shaft diameter $(\mathrm{mm})$. The tolerance of the study treatment was evaluated by recording and monitoring any adverse event at baseline and on each following visit. Moreover, at the end of the study (T1) and at the Follow Up Visit (T2), each subject was asked to fill a questionnaire regarding the efficacy of the study treatment on daily hair loss, volume, on hair general aspect (hair thicker and stronger), on hair growth speed and the product tolerance (score: negative, medium, good, excellent). No hormonal data have been collected from analysed subjects.

\section{Statistical analysis}

Comparisons between two individual visits were performed by means of two-sample-t-test. P-values less than 0.05 were considered clinically significant.

\section{Results}

A total of 60 subjects ( 32 men and 28 women; women) were enrolled and received treatment (Table 1). The two groups were generally similar in terms of baseline characteristics.

As determined by Trichoscan digital image analysis, after 3 months of treatment (T1) TR-M-PRP plus produced a mean increase of 15.3 hairs (T0 TR-M-PRP plus vs T1 TR-M-PRP plus: $\mathrm{p}<0,0001$ ), while PLACEBO produced a decrease of 11.1 hairs (Table 2).

TR-M-PRP plus efficacy was more evident at follow-up examination (+25.2; T0 TR-M-PRP plus vs T2 TR-M-PRP plus: $\mathrm{p}<0,0001)$; no significative changes between $\mathrm{T} 1$ and $\mathrm{T} 2$ were reported in the PLACEBO group.

TR-M-PRP plus treatment also led to a progressive increase in mean anagen hair count over 3 months ( $p<0,0001$, Table 2$)$. A further significative increase was also reported at the follow-up examination $(\mathrm{p}<0,0001$, Table 2$)$. On the contrary treatment with PLACEBO led to a decrease in mean anagen hair count $(\mathrm{p}<0,0001$, Table 2$)$.

Table 1. Baseline characteristics of subjects randomized

\begin{tabular}{|c|c|c|}
\hline & TR-M-PRP plus (n=30) & PLACEBO $(n=30)$ \\
\hline Age (mean \pm SE) & $39.42 \pm 3.96$ & $31.84 \pm 1.80$ \\
\hline \multicolumn{3}{|l|}{ Baseline hair count $(\text { mean } \pm \mathrm{SD})^{\mathrm{a}}$} \\
\hline Total hair count & $194.27 \pm 4.0$ & $199.25 \pm 3.00$ \\
\hline Anagen & $129.2 \pm 5.0$ & $120.85 \pm 2.95$ \\
\hline$\%$ Anagen & $66.51 \pm 4.0$ & $60.65 \pm 4.75$ \\
\hline Anagen/telogen ratio & $1.98 \pm 0.2$ & $1.54 \pm 0.15$ \\
\hline Miniaturization (\%) & $48.34 \pm 3.0$ & $50.97 \pm 3.13$ \\
\hline Hair Mass Index (HMI) & $1.98 \pm 0.2$ & $1.54 \pm 0.15$ \\
\hline Hair shaft diameter (mm) & $0.51 \pm 0.1$ & $0.57 \pm 0.06$ \\
\hline
\end{tabular}

a Measured in a $1{ }^{-\mathrm{cm} 2}$ circle 
TR-M-PRP plus treatment led to a statistically significant improvement of anagen/telogen ratio after 3 months of treatment which resulted in further incremented at follow-up examination $(\mathrm{p}<0,0001$, Table 2$)$. Also in this evaluation, a decrease was reported in the PLACEBO group $(\mathrm{p}<0,00)$

Figure 1 reported data on the percentage of miniaturization for TR-M-PRP plus and PLACEBO group, respectively. We noticed a significative decrease in hair miniaturization \% in TR-M-PRP plus after 3 months of treatment compared to the PLACEBO group $(40.40 \pm 1.85$ vs $49.58 \pm 2.74)(\mathrm{p}<0,0001)$. The effect derived from TR-M-PRP plus treatment is more evident at follow-up examination $(23.61 \pm 1.85$ vs $49.27 \pm 2.72)(\mathrm{p}<0,0001)$.

Following treatment with TR-M-PRP plus (Figure 2) HMI was significantly increased $(162.27 \pm 4.57$ to $187.90 \pm 6.07 ; \mathrm{p}<0.0001)$ and the effect maintained at follow up $(198.17 \pm 6.05$; T2 vs T1: $\mathrm{p}<0.0001)$ (Figure 2). This increase was statistically significant when compared with PLACEBO group (T1 TR-M-PRP plus vs T1 PLACEBO: $\mathrm{p}<0,0001$; T2 TR-M-PRP plus vs T2 PLACEBO: $\mathrm{p}<0,0001)$. In PLACEBO group no significative change was reported. No adverse event related to the study products occurred during the trial and the efficacy and good tolerance of the study products was also confirmed by the volunteers judgment.

\section{Discussion}

AGA is the most reported form of hair loss [26] which main symptoms include miniaturization of the hair follicle [27,28]. Also, hair growth cycle is altered in AGA subjects [29]. Current research is highlighting the regulatory role of many substances, especially growth factors, on the hair follicle cycle [30].

Growing interest is focused on polypeptide growth factors. They can target many physiological processes such as cell proliferation, protein synthesis, inflammation and pigmentation [31].

More recently, polypeptide growth factors involved in the regulation of hair growth cycle have been established [32,33]. Thanks to the advent of modern biotechnology polypeptides mimicking

Table 2. Hair count data $(\operatorname{mean} \pm \mathrm{SD})$

\begin{tabular}{|c|c|c|c|c|c|c|}
\hline & \multicolumn{3}{|c|}{ TR-M-PRP plus (n=30) } & \multicolumn{3}{|c|}{$\operatorname{PLACEBO}(\mathrm{n}=30)$} \\
\hline & Baseline (T0) & T1 & $\mathbf{T 2}$ & Baseline (T0) & T1 & $\mathbf{T 2}$ \\
\hline Total hair count & $194.3 \pm 4.0$ & $204.2 \pm 3.2$ & $219.5 \pm 3.3$ & $199.3 \pm 3.0$ & $188.3 \pm 3.3$ & $187.30 \pm 3.4$ \\
\hline Anagen hair count & $129.2 \pm 5.0$ & $142.5 \pm 2.8$ & $162.6 \pm 2.9$ & $120.8 \pm 2.9$ & $110.3 \pm 3.9$ & $116.5 \pm 3.55$ \\
\hline Telogen hair count ${ }^{\mathrm{a}}$ & $65.1 \pm 4.45$ & $61.6 \pm 2.6$ & $56.8 \pm 2.5$ & $78.4 \pm 3.8$ & $77.9 \pm 3.9$ & $70.80 \pm 3.45$ \\
\hline Anagen/telogen ratio ${ }^{b}$ & $1.9 \pm 0.2$ & $2.3 \pm 0.24$ & $2.9 \pm 0.2$ & $1.5 \pm 0.1$ & $1.4 \pm 0.3$ & $1.64 \pm 0.16$ \\
\hline
\end{tabular}

${ }^{\mathrm{a}}$ Telogen hair count $=($ Total hair count $)-($ Anagen hair count $) .{ }^{\mathrm{b}}$ Anagen/telogen ratio=mean $($ anagen hair count/telogen hair count $)$

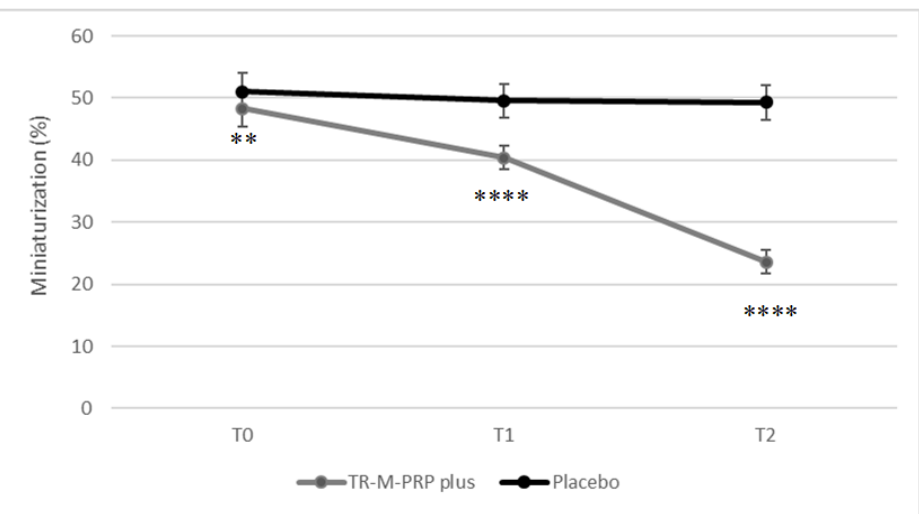

Figure 1. \% of hair miniaturization as measured by TrichoScan software (v. 3.0). Data (miniaturization \%) are expressed as mean \pm SD of 30 subjects for the group, at baseline (T0), after 3 months of treatment (T1) and at follow-up (T2). **=p $<0.01 ; * * * *=\mathrm{p}<0.001$

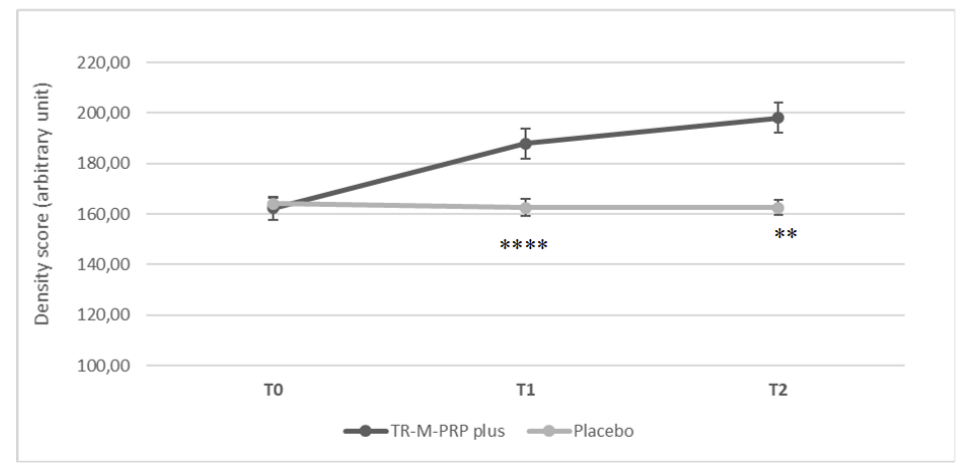

Figure 2. Hair Mass Index (HMI) as measured by HairCheck ${ }^{\circledR}$ measuring system. Data (density score) are expressed as mean \pm SD of 30 subjects for the group, at baseline (T0), after 3 months of treatment (T1) and at follow-up (T2). ****=p $<0.001$ 
growth factors have been developed [34], opening to new therapeutic approaches also in the dermatological field, including hair growth disorder.

The aim of the present work was to show the efficacy of TR-M-PRP plus, a cosmetic product mimicking autologous PRP composition in term of growth factors on subjects affected by AGA.

Efficacy of treatment with autologous PRP has largely been demonstrated both in AA [11,19] and AGA [21,22] subjects. More recently we published a work on the efficacy of TR-M-PRP plus in the treatment of AA [23].

In the present work, we tested the same cosmetic formulation on AGA subjects. The results show significant changes in the objective parameters which were tested to evaluate the effectiveness of the treatment.

TR-M-PRP plus treatment increased the amount and percentage of anagen hair progressively from baseline over 3 months of treatments and at follow-up examination. Also, a progressive increase of anagen/ telogen ratio has been reported. On the opposite, in PLACEBOtreated group anagen hair diminished during the study, consistent with follicular miniaturization.

The efficacy of the treatment on the promotion of anagen phase was further validated by a significant decrease of follicular miniaturization and the increase both of HMI and Hair shaft diameter. Therefore, all investigated subjects reported a good tolerance of the treatment and no side effects. This is in line with recent evidence on the stability and safety of biomimetic polypeptides [35].

The above-reported efficacy of TR-M-PRP plus could be ascribed to the effect of biomimetic peptides used (octapeptide-2, copper tripeptide-1, Oligopeptide-20, acetyl decapeptide-3) on cell proliferation stimulation. Treatment efficacy is further enhanced by the anti-inflammatory effect exerted by lactoferrin [36], enhancing of cellular energy by lactoglobulin [37] and clock-regulatory activity of melatonin [38].

Taking together, our data strongly suggest that the treatment with a cosmetic product mimicking autologous PRP, via biomimetic polypeptides, actively increases growing anagen hair.

\section{Conclusion}

In conclusion, our study confirms, for the first time, the clinical efficacy of a cosmetic product containing biomimetics peptides (TR-M-PRP plus) mimicking autologous PRP for the treatment of AGA. Treatment result has well tolerated and no adverse events were reported.

\section{Disclosure statement}

R.F. and S.E. serve as a consultant for Giuliani S.p.A. P.D. and M.B. are employed by Giuliani S.p.A.

\section{Funding}

This study was supported by the Giuliani S.p.A.

\section{References}

1. Ellis JA, Sinclair R, Harrap SB (2002) Androgenetic alopecia: Pathogenesis and potential for therapy. Expert Rev Mol Med 4: 1-11. [Crossref]

2. Rogers NE, Avram MR (2008) Medical treatments for male and female pattern hair loss. J Am Acad Dermatol 59: 547-566. [Crossref]
3. Piraccini BM, Alessandrini A (2014) Androgenetic alopecia. G Ital Dermatol Venereol 149: 15-24. [Crossref]

4. Deloche $\mathrm{C}$, de Lacharrière $\mathrm{O}$, Misciali C (2004) Histological features of peripilar signs associated with androgenetic alopecia. Arch Dermatol Res 295: 422-428. [Crossref]

5. Hernandez BA (2004) Is androgenic alopecia a result of endocrine effects on the vasculature? Med Hypotheses 62: 438-441. [Crossref]

6. Reygagne P, de Lacharriere O Alopecia (2005) In: Bouillon C, Wilkinson J [Eds.] The Science of Hair Care. 2nd Edition. New York: Taylor \& Francis. pp: 559-582.

7. Messenger AG, Sinclair R (2006) Follicular miniaturization in female pattern hair loss: clinicopathological correlations. Br J Dermatol 155: 926-930. [Crossref]

8. Williamson D, Gonzalez M, Finlay AY (2001) The effect of hair loss on quality of life. J Eur Acad Dermatol Venereol 15: 137-139. [Crossref]

9. Phillips TG, Slomiany WP, Allison R (2017) Hair loss: Common causes and treatment Am Fam Physician 96: 371-378. [Crossref]

10. Rossi A, Anzalone A, Fortuna MC, Caro G, Garelli V, et al. (2016) Multi-therapies in androgenetic alopecia: Review and clinical experiences. Dermatol Ther 29: 424-432. [Crossref]

11. Cole JP, Cole MA, Insalaco C, Cervelli V (2017) Alopecia and platelet-derived therapies. Stem Cell Investig 4: 88. [Crossref]

12. Cervelli V, Garcovich S, Bielli A (2014) The effect of autologous activated platele rich plasma (AA-PRP) Injection on pattern hair loss: Clinical and histomorphometric evaluation. BioMed Res Int 9: 2014.

13. Li ZJ, Choi HI, Choi DK, Sohn KC, Im M, et al. (2012) Autologous platelet-rich plasma: a potential therapeutic tool for promoting hair growth. Dermatol Surg 38: 1040-1046. [Crossref]

14. Ferrari M, Zia S, Valbonesi M, Henriquet F, Venere G, et al. (1987) A new technique for hemodilution, preparation of autologous platelet-rich plasma and intraoperative blood salvage in cardiac surgery. Int J Artif Organs 10: 47-50. [Crossref]

15. Anitua E, Sánchez M, Nurden AT, Nurden P, Orive G, et al. (2006) New insights into and novel applications for platelet-rich fibrin therapies. Trends Biotechnol 24: 227-234. [Crossref]

16. Foster TE Puskas BL, Mandelbaum BR, Gerhardt MB, Rodeo SA (2009) Platelet-rich plasma: from basic science to clinical applications. Am J Sports Med 37: 2259-2272. [Crossref]

17. Rossano F, Di Martino S, Iodice L, Di Paolo M, Misso S, et al. (2017) Correlation between individual inflammation genetic profile and platelet rich plasma efficacy in hair follicle regeneration: A pilot study reveals prognostic value of IL-1a polymorphism. Eur Rev Med Pharmacol Sci 21: 5247-5257. [Crossref]

18. Giordano S, Romeo M, di Summa P, Salval A, Lankinen P (2018) A Meta-analysis on evidence of platelet-rich plasma for androgenetic alopecia. Int J Trichology 10: 1-10. [Crossref]

19. Sorbellini E, Trink A, Rinaldi F (2011) Experimental clinical assessment of the use of platelet-rich plasma in dermatology and rationale for its use in the treatment of nonscarring alopecia. 35th La Medicina Estetica p, 43.

20. Trink A, Sorbellini E, Bezzola P (2013) A randomized, double blind, placebo- and active-controlled, half-head study to evaluate the effects of platelet-rich plasma on alopecia areata. Br J Dermatol 169: 690-694. [Crossref]

21. Alves R, Grimalt R (2016) Randomized placebo-controlled, doubleblind, half-head study to assess the efficacy of platelet-rich plasma on the treatment of androgenetic alopecia. Dermatol Surg 42: 491-497.

22. Mapar MA, Shahriari S, Haghighizadeh MH (2016) Efficacy of platelet-rich plasma in the treatment of androgenetic (male-patterned) alopecia: a pilot randomized controlled trial. J Cosmet Laser Ther 18: 452-455. [Crossref]

23. Rinaldi F, Marzani B, Pinto D, Sorbellini E. Randomized (2018) Controlled trial on a PRP-like cosmetic, biomimetic peptides based, for the treatment of alopecia areata. $J$ Dermatolog Treat 4: 1-6.

24. Wikramanayake TC, Mauro LM, Tabas IA, Chen AL, Llanes IC. et al. (2012) A clinical tool for assessing the progression and treatment response of alopecia. Int $J$ Trichology 4: 259-264. [Crossref]

25. Marzani B, Pinto D, Sorbellini E, Rinaldi F (2018) New multi-targeting strategy in hair growth promotion: in vitro and in vivo studies. G Ital Dermatol Venereol 153 : 338-343. [Crossref] 
26. Piraccini BM, Alessandrini A (2014) Androgenetic alopecia. G Ital Dermatol Venereol 149: 15-24. [Crossref]

27. Hoffmann R, Happle R (2000) Current understanding of androgenetic alopecia. Part I etiopathogenesis. Eur J Dermatol 10: 319-327. [Crossref]

28. Hoffmann R, Happle R (2000) Current understanding of androgenetic alopecia. Part II: clinical aspects and treatment. Eur J Dermatol 10: 410-417. [Crossref]

29. Courtois M, Loussouarn G, Hourseau C, Grollier JF (1994) Hair cycle and alopecia. Skin Pharmacol 7: 84-89. [Crossref]

30. Brajac I, Vicic M, Periša D, Kaštelan M (2014) Human hair follicle: An update on biology and perspectives in hair growth disorders treatment. Hair Ther Transplant 4 115 .

31. Deuel TF (1987) Polypeptide growth factors: roles in normal and abnormal cell growth Annu Rev Cell Biol. 3: 443-92. [Crossref]

32. Takakura N, Yoshida H, Kunisada T (1996) Involvement of platelet-derived growth factor receptor-alpha in hair canal formation. J Invest Dermatol 107: 770-777. [Crossref]
33. Jain R, De-Eknamkul W (2014) Potential targets in the discovery of new hair growth promoters for androgenic alopecia. Expert Opin Ther Targets 18: 787-806. [Crossref]

34. Reddy B, Jow T, Hantash BM (2012) Bioactive oligopeptides in dermatology: Part I. Exp Dermatol 21: 563-568. [Crossref]

35. Zhang K, Letham DS, John PC (1996) Cytokinin controls the cell cycle at mitosis by stimulating the tyrosine dephosphorylation and activation of p34cdc2-like H1 histone kinase. Planta 200: 2-12.

36. Tai CS, Chen YY, Chen WL (2016) $\beta$-lactoglobulin influences human immunity and promotes cell proliferation. BioMed Res Int 2016: 1. [Crossref]

37. Geyfman M, Andersen B (2010) Clock genes, hair growth and aging. Aging (Albany NY) 2: 122-128. [Crossref]

38. Gazitaeva ZI, Drobintseva AO, Chung Y, Polyakova VO, Kvetnoy IM (2017) Cosmeceutical product consisting of biomimetic peptides: antiaging effects in vivo and in vitro. Clin Cosmet Investig Dermatol 10: 11-16.

Copyright: (O2019 Rinaldi F. This is an open-access article distributed under the terms of the Creative Commons Attribution License, which permits unrestricted use, distribution, and reproduction in any medium, provided the original author and source are credited. 